\title{
COMPOSIÇÃO FLORÍSTICA E ESPECTRO BIOLÓGICO NA ESTAÇÃO ECOLÓGICA DE SANTA BÁRBARA, ESTADO DE SÃO PAULO, BRASIL ${ }^{1}$
}

\author{
João Augusto Alves Meira Neto ${ }^{2}$, Fernando Roberto Martins ${ }^{3}$ e Gilmar Edilberto Valente ${ }^{4}$
}

\begin{abstract}
RESUMO - O conhecimento da flora herbáceo-subarbustiva, juntamente com o da flora lenhosa, auxilia a determinação dos padrões florísticos e permite descrever o espectro biológico com conseqüentes inferências sobre a atuação de fatores ambientais e históricos na vegetação. Considerando que poucos trabalhos se aprofundaram no estudo da flora herbáceo-subarbustiva de Cerrado, embora esta seja mais rica que a lenhosa, objetivouse estudar a composição e os padrões florísticos das floras herbáceo-subarbustiva e lenhosa da Estação Ecológica de Santa Bárbara (EESB) $\left(22^{\circ} 46^{\prime} 30^{\prime \prime}\right.$ a $22^{\circ} 50^{\prime} 30^{\prime \prime} \mathrm{S}$ e $49^{\circ} 10^{\prime} 30^{\prime \prime}$ a $49^{\circ} 15^{\prime} 30^{\prime \prime} \mathrm{W}, 600$ a $680 \mathrm{~m}$ de altitude), Município de Águas de Santa Bárbara, Estado de São Paulo. Visou-se, ainda, determinar o espectro biológico para efetuar análises comparativas das diferentes fitofisionomias de Cerrado dessa Unidade de Conservação. Foram encontradas 314 espécies na EESB, sendo 285 em Cerrado sensu lato. As famílias mais ricas em número de espécies foram Asteraceae, Leguminosae, Myrtaceae e Poaceae. Há uma constante ocorrência de Asteraceae, Leguminosae e Poaceae entre as famílias mais ricas, concordando com o observado nos estudos florísticos de Cerrado que incluíram os estratos lenhoso e herbáceo-subarbustivo. O espectro biológico corroborou os padrões anteriormente descritos para o Cerrado sensu lato, exceto pela maior expressão de caméfitas em relação às hemicriptófitas nas fisionomias campestres da EESB, o que pode ser efeito da proteção ao fogo nessa Unidade de Conservação.
\end{abstract}

Palavras-chave: Cerrado, formas de vida e padrões florísticos.

\section{FLORISTIC COMPOSITION AND BIOLOGICAL SPECTRA IN SANTA BARBARA ECOLOGICAL STATION, BRAZIL}

\begin{abstract}
Only few surveys were carried out on woody and ground layer floras of the Brazilian Cerrado. The objective of this survey was to investigate richness, floristic patterns and biological spectra of different phytophysiognomies on both strata at Santa Bárbara Ecological Station (EESB). EESB is located in the Municipality of Águas de Santa Bárbara, São Paulo State, Brazil (22 $46^{\prime} 30^{\prime \prime}$ to $22^{\circ} 50^{\prime} 30^{\prime \prime} S$ and $49^{\circ}$ $10^{\prime} 30^{\prime \prime}$ to $49^{\circ} 15^{\prime} 30^{\prime \prime} \mathrm{W}, 600$ to $680 \mathrm{~m}$ high). A total of 14 angiospermic species were found, 285 of these in Cerrado sensu lato physiognomies. The richest families in species number were Asteraceae, Fabaceae, Myrtaceae and Poaceae. Asteraceae, Fabaceae and Poaceae were the richest families similar to other Cerrado's sites. The EESB biological spectra have patterns similar to other Cerrados, but with one change: chamaephytes were richer than hemycryptophytes in the open savannic physiognomies of EESB. Fire protection may be the reason of this alteration.
\end{abstract}

Keywords: Cerrado vegetation, Brazilian savanna and Raunkiaer's life forms.

\footnotetext{
${ }^{1}$ Recebido em 12.05.2006 e aceito para publicação em 01.12.2006.

${ }^{2}$ Departamento de Biologia Vegetal da Universidade Federal de Viçosa. E-mail: <meiraneto@ ufv.br>.

${ }^{3}$ Departamento de Botânica da Universidade Estadual de Campinas. E-mail: <fmartins@ unicamp.br>.

${ }^{4}$ Herbário da Universidade Federal de Viçosa. E-mail: <gvalente@ufv.br>.
} 


\section{INTRODUÇÃO}

Os estudos florísticos e fitossociológicos em florestas de todo o mundo geralmente enfatizam o componente arbóreo, que é o principal detentor da biomassa florestal e se destaca pela importância econômica (MEIRA NETO e MARTINS, 2003). Pelos mesmos motivos, o componente lenhoso da vegetação de Cerrado foi mais estudado, e são poucos os trabalhos que deram atenção tanto ao componente lenhoso quanto ao herbáceo-subarbustivo. Os estudos de Mantovani (1987), Mantovani e Martins (1993), Mendonça et al. (1998), Batalha e Mantovani (2000, 2001) e Batalha e Martins (2002, 2004) foram importantes contribuições para um conhecimento florístico de ambos os componentes da flora do Cerrado.

Trabalhos clássicos sobre o Cerrado apontam a existência de duas floras independentes e concorrentes, a herbáceo-subarbustiva e a arbustivo-arbórea, ou lenhosa (RIZZINI, 1963; COUTINHO, 1978, 2002). A flora herbáceo-subarbustiva é de especial importância para a compreensão da riqueza da flora do Cerrado, por possuir um número de espécies que pode ser de três a mais de quatro vezes maior que o de espécies lenhosas (FILGUEIRAS, 2002).

Warming (1973) realizou um dos trabalhos pioneiros de Ecologia Vegetal e o primeiro estudo em Cerrado na segunda metade do século 19. Neste trabalho, Warming considerou a família Compositae (Asteraceae) a mais rica nos Cerrados de Lagoa Santa, MG. Os levantamentos florísticos que estudaram as duas floras em conjunto indicaram a existência de um possível padrão, em que as famílias Asteraceae, Leguminosae e Poaceae aparecem como as mais ricas no Cerrado. Além da confirmação dos padrões na categoria taxonômica de famílias, a grande riqueza do Cerrado é uma forte justificativa para estudos florísticos que considerem todos os estratos e não apenas o arbustivo-arbóreo (MENDONÇA et al., 1998; CASTRO et al., 1999; BATALHAe MANTOVANI, 2001). É pela gande riqueza, e por ser uma das maiores fronteiras agrícolas do mundo, que o Cerrado é considerado uma das 25 prioridades de conservação de biodiversidade do planeta (MYERS et al., 2000).

O conhecimento sobre a composição florística permite o estudo das formas de vida das espécies presentes em determinado ambiente. As formas de vida de Raunkiaer de uma fitocenose constituem seu espectro biológico. Os estudos sobre espectro biológico no
Domínio do Cerrado evidenciaram padrões nas distribuições das formas de vida em diferentes fitofisionomias (MANTOVANI, 1983; MANTOVANI e MARTINS, 1993; BATALHA et al.., 1997; BATALHA e MANTOVANI, 2001; BATALHA e MARTINS, 2002, 2004). Os espectros biológicos têm mostrado predominância de fanerófitas em Cerrado sensu stricto e Cerradão e predominância de hemicriptófitas em campo cerrado, campo sujo e campo limpo. Os padrões encontrados possibilitaram inferências sobre fatores ambientais e históricos que influenciam essas comunidades, como clima, gradientes pedológicos, fogo ou pastejo.

Este trabalho teve como objetivo contribuir com o conhecimento das floras lenhosa e herbáceosubarbustiva do Cerrado da Estação Ecológica de Santa Bárbara (EESB), Município de Águas de Santa Bárbara, SP, para (i) avaliar sua riqueza, (ii) estabelecer comparações florísticas diretas em nível de famílias para a flora de Cerrado e (iii) estabelecer comparações do espectro biológico entre as diferentes fitofisionomias estudadas e entre diferentes áreas de Cerrado, inferindo sobre fatores ambientais e históricos da EESB.

\section{MATERIAL E MÉTODOS}

A Estação Ecológica de Santa Bárbara (EESB), criada através do Decreto Estadual 22.337, de 07/06/1984, possui uma área de 2.715,5 há, no Município de Águas de Santa Bárbara, Estado de São Paulo (BARRETO, 1985). AEstação Ecológica (Figura 1) pertence ao Instituto Florestal do Estado de São Paulo e situa-se entre as coordenadas $22^{\circ} 46^{\prime} 30^{\prime \prime}$ a $22^{\circ} 50^{\prime} 30^{\prime \prime} \mathrm{S}$ e $49^{\circ} 10^{\prime} 30^{\prime \prime}$ a $49^{\circ} 15^{\prime} 30^{\prime \prime} \mathrm{W}$, próximo do limite sul de distribuição de Cerrado, com altitudes variando entre 600 e $680 \mathrm{~m}$.

O clima é do tipo Cwa de Koeppen, com as temperaturas médias do mês mais quente (janeiro) entre 23 e $24 \mathrm{C}$ e as temperaturas médias do mês mais frio (julho) em torno dos $16 \mathrm{C}$. A precipitação média anual fica entre 1.100 e $1.300 \mathrm{~mm}$, com invernos secos e verões chuvosos (SETZER, 1966).

Os solos, classificados segundo a Embrapa (1999), determinam diferentes fitofisionomias do Cerrado. Latossolo Vermelho e Latossolo Vermelho-Amarelo ocorrem nos locais com Cerradão e Cerrado sensu stricto. Neossolo Quartzarênico aparecem nos locais com campo cerrado e campo sujo. Alissolo ocorre onde se distribui o campo limpo úmido (VENTURA et al.,1965/1966). 


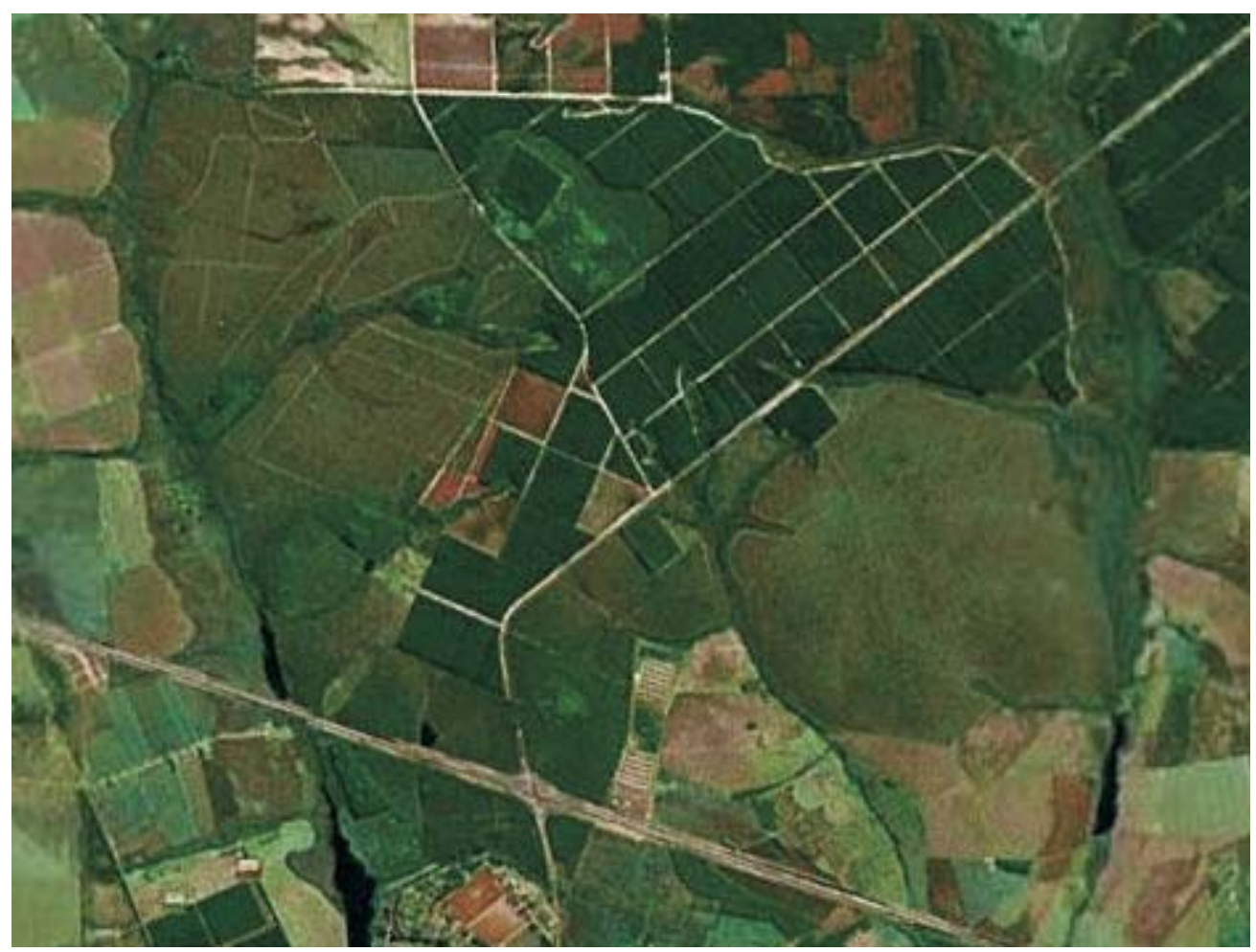

Figura 1 - Imagem da Estação Ecológica de Santa Bárbara (EESB) do Instituto Florestal do Estado de São Paulo orientada com o norte no alto. Seus limites ao Leste e ao Oeste são córregos afluentes do rio Pardo. Ao norte, uma estrada com direção leste-oeste separa os talhões de Pinus e Eucalyptus da EESB (no alto ao centro e à direita na imagem) e o Cerrado da EESB (no alto à esquerda na imagem), de propriedades particulares. No extremo sul, os limites da EESB na mancha urbana de Águas de Santa Bárbara abaixo da rodovia SP 280 - Castelo Branco, que corta a imagem com direção leste-sudeste a oeste-noroeste. Ao sul-sudeste, os limites são com campos de cultivo e pastagens, acima da SP 280. Dentro de seus limites, as áreas poligonais são talhões de Pinus e Eucalyptus. As demais áreas são de Cerrado sensu lato, do verde mais escuro ao sul (Cerrado sensu stricto) ao verde menos escuro (campos), exceto nas linhas de drenagem, onde ocorrem Matas de Galeria.

Figure 1 - Image of Santa Barbara Ecological Station (EESB) - São Paulo Forest Institute, with the north at the top. The EESB east and west limits are streams that flow to the Pardo River. In the north, a road separates the EESB's Cerrado and EESB's Pinus and Eucalyptus plantation from the private areas. At bottom, below the SP280 highway, the limit is Aguas de Santa Barbara town. Other EESB limits neighboring crop fields and pastures. Deep green polygonal areas inside EESB are Pinus and Eucalyptus plantations. Other green areas of Cerrado are denser than deeper, except along the streams, where gallery forests grow.

Para o levantamento florístico foram feitas coletas de material em fase reprodutiva durante 24 meses, de Janeiro de 1989 a Janeiro de 1990. As coletas foram feitas mediante caminhadas na vegetação e por meio da aplicação de 30 parcelas de $10 \times 20 \mathrm{~m}$, alocadas sistematicamente no Cerrado sensu stricto, na extremidade sul da EESB, ao sul da rodovia SP 280 (Figura 1). As exsicatas foram identificadas no nível mais exclusivo possível, através de literatura taxonômica, consultas a herbários e a especialistas. Em seguida, foram incorporadas aos herbários da UNICAMP (UEC), Bento
Pickel, do Instituto Florestal do Estado de São Paulo (SPSF) e da Universidade Federal de Viçosa (VIC). A lista de espécies foi organizada pelo sistema APG II (APG, 2003).

Para a identificação dos tipos fisionômicos da vegetação da EESB foram utilizados os trabalhos de Goodland (1971) e Eiten (1990).

O espectro biológico das formas de vida de Raunkiaer foi elaborado de acordo com a chave de identificação de Mueller-Dombois e Ellenberg (1974). 


\section{RESULTADOS E DISCUSSÃO}

Foram relacionadas 314 espécies pertencentes a 84 famílias de angiospermas que ocorrem na Estação Ecológica de Santa Bárbara (EESB). Dessas espécies, 285 predominam nas fitofisionomias de Cerrado sensu lato (Quadro 1). Embora expressivo, o número de espécies encontradas no Cerrado sensu lato da EESB é menor que o encontrado em outras Unidades de Conservação em Cerrado, como o Parque Nacional das Emas (GO), com 601 espécies; a Reserva Biológica de Moji-Guaçu
(SP), com 532 espécies; e a Reserva Biológica de MojiGuaçu (SP), com 360 espécies (MANTOVANI e MARTINS 1993; BATALHA e MANTOVANI, 2001; BATALHA e MARTINS 2002, 2004;). Uma efetiva conservação dos 2.715 ha da EESB protege o Cerrado sensu lato local, que ainda apresenta grande parte de sua riqueza original, com expressivo número de espécies que representam $47 \%$ do total encontrado no Parque Nacional das Emas, uma Unidade de Conservação com uma área 49 vezes maior (133.000 ha).

Quadro 1 - Espécies coletadas na Estação Ecológica de Santa Bárbara, Município de Águas de Santa Bárbara, Estado de São Paulo. Formas de vida: FAN - fanerófita, CAM - caméfita, HEM - hemicriptófita, GEO = geófita, TER $=$ terófita, $\mathrm{LIA}=$ liana, $\mathrm{SPV}=$ semiparasita vascular e $\mathrm{SAP}=$ saprófita vascular. Fisionomias: $\mathrm{C}$ - cerradão, $\mathrm{CE}$ Cerrado sensu stricto, CC - campo cerrado, CS campo sujo, CL - campo limpo úmido e M - mata de galeria. Número de coleta de J.A.A.Meira Neto

Table 1 - Collected species in Águas de Santa Bárbara Ecological Station (EESB), SP, Brazil. Life forms: FAN-fanerophyte, CAM - chamaephyte, HEM - hemycryptophyte, GEO - cryptophyte, TER - terophyte, LIA - liana, SPV - vascular semi-parasite, SAP - vascular saprophyte. Physiognomies (fisionomias): C-cerradão (closed savanna approaching a forest), CE Cerrado sensu stricto; CC - campo cerrado (open savanna); CS campo sujo (grassland with some shrubs); CL - campo limpo úmido (wet clean grass fields); M-gallery forest. Accession number by J.A.A.Meira Neto

\begin{tabular}{|c|c|c|c|}
\hline FAMÍLIA/ESPÉCIE & $\begin{array}{l}\text { FORMA } \\
\text { DE VIDA }\end{array}$ & FISIONOMIA & $\begin{array}{l}\text { NÚMERO DE } \\
\text { COLETA }\end{array}$ \\
\hline \multicolumn{4}{|l|}{ ACANTHACEAE } \\
\hline Ruellia geminiflora Kunth & CAM & $\mathrm{CCCS}$ & $(676,492)$ \\
\hline Ruellia sp. & CAM & CS & $(706)$ \\
\hline ALISMATACEAE & & & \\
\hline $\begin{array}{l}\text { Echinodorus sp. } \\
\text { AMARANTHACEAE }\end{array}$ & HEM & M & $(644)$ \\
\hline Gomphrena macrocephala St. Hil. & HEM & CE CC CS & $(714,502)$ \\
\hline Pfaffia helichrysoides (Mart.) Kuntze & CAM & CS & $(657)$ \\
\hline $\begin{array}{l}\text { Pfaffia paniculata (Mart.) Kuntze } \\
\text { ANACARDIACEAE }\end{array}$ & LIA & $\mathrm{C}$ & $(619)$ \\
\hline Anacardium humile A. St.-Hil. & GEO & $\mathrm{CE} \mathrm{CC} \mathrm{CS}$ & (759) \\
\hline Lithraea molleoides (Vell.) Engl. & FAN & CE M & (719) \\
\hline Tapirira guianensis Aubl. & FAN & C CE M & $(720,477)$ \\
\hline \multicolumn{4}{|l|}{ ANNONACEAE } \\
\hline Annona dioica A. St.-Hil. & FAN & $\mathrm{CC}$ & $(758,473,504)$ \\
\hline Annona coriacea Mart. & FAN & C CE CC & $(717)$ \\
\hline Annona crassiflora Mart. & FAN & CE CC & $(718)$ \\
\hline Duguetia furfuracea (A.St.-Hil.) Benth. \& Hook. f & FAN & CE CC & $(557)$ \\
\hline $\begin{array}{l}\text { Guatteria nigrescens } \text { Mart. } \\
\text { APIACEAE }\end{array}$ & FAN & $\mathrm{C}$ & $(459)$ \\
\hline $\begin{array}{l}\text { Eryngium koehneanum Urb. } \\
\text { APOCYNACEAE }\end{array}$ & HEM & CS & $(501)$ \\
\hline Aspidosperma tomentosum Mart. & FAN & C CE CC & (716) \\
\hline Blepharodon bicuspidatum E. Fourn. & LIA & $\mathrm{CE}$ & $(555)$ \\
\hline Condylocarpon rauwolfiae (A. DC.) Müll. Arg. & LIA & M & $(545)$ \\
\hline Forsteronia thyrsoidea (Vell.) Müll. Arg. & LIA & $\mathrm{CE}$ & $(401)$ \\
\hline
\end{tabular}

R. Árvore, Viçosa-MG, v.31, n.5, p.907-922, 2007 
Quadro 1-Cont.

Table 1 -Cont.

\begin{tabular}{|c|c|c|c|}
\hline FAMÍLIA/ESPÉCIE & $\begin{array}{l}\text { FORMA } \\
\text { DE VIDA }\end{array}$ & FISIONOMIA & $\begin{array}{l}\text { NÚMERO DE } \\
\text { COLETA }\end{array}$ \\
\hline \multicolumn{4}{|l|}{ APOCYNACEAE } \\
\hline Mandevilla velutina K.Schum. & HEM & $\mathrm{CE}$ & $(478)$ \\
\hline Peschiera fuchsiaefolia (A. DC.) Miers & FAN & CE M & (549) \\
\hline \multirow{2}{*}{\multicolumn{4}{|c|}{ ARALIACEAE }} \\
\hline & & & \\
\hline \multicolumn{4}{|l|}{ ARECACEAE } \\
\hline Attalea geraensis Barb. Rodr. & HEM & CE CC & $(417)$ \\
\hline Geonoma gamiova Barb. Rodr. & FAN & M & $(648)$ \\
\hline Syagrus loefgrenii Glassman & HEM & C CE CC CS & $(415,497,715)$ \\
\hline Syagrus romanzoffiana (Cham.) Glassman & FAN & $\mathrm{C}$ & $(752)$ \\
\hline \multicolumn{4}{|l|}{ ASTERACEAE } \\
\hline Achyrocline satureioides (Lam.) DC. & CAM & $\mathrm{CE}$ & $(359,518)$ \\
\hline Aspilia montevidensis (Spreng.) Kuntze & CAM & CS & $(669)$ \\
\hline Aspilia reflexa Baker & CAM & CE CL & $(538,694)$ \\
\hline Baccharis semiserrata DC. & FAN & CE CC CS & $(410)$ \\
\hline Baccharis trimera (Less.) DC. & CAM & CL & $(757)$ \\
\hline Bidens gardneri Baker & CAM & CS & $(526)$ \\
\hline Calea platylepis Sch. Bip. & CAM & $\mathrm{CCCS}$ & $(655)$ \\
\hline Chaptalia integerrima (Vell.) Burkart & HEM & $\mathrm{CCCS}$ & $(661)$ \\
\hline Dasyphyllum orthacanthum (DC.) Cabrera & FAN & M & $(701)$ \\
\hline Eremanthus sphaerocephalus (DC.) Baker & CAM & $\mathrm{CE}$ & $(381,405,419,433)$ \\
\hline Eupatorium ascendens Sch. Bip. ex Baker & CAM & CS & $(524)$ \\
\hline Eupatorium barbacense Hieron. & FAN & $\mathrm{CE}$ & $(356,489)$ \\
\hline Eupatorium betonicaeforme (DC.) Baker & FAN & CS & $(580)$ \\
\hline Eupatorium gaudichaudianum DC. & FAN & $\mathrm{CE}$ & $(515)$ \\
\hline Eupatorium ivaefolium $\mathrm{L}$. & CAM & CL & $(565)$ \\
\hline Eupatorium laevigatum Lam. & FAN & $\mathrm{CE}$ & $(482,513)$ \\
\hline Eupatorium squalidum DC. & CAM & $\mathrm{CE}$ & $(355)$ \\
\hline Gochnatia barrosii Cabrera & FAN & $\mathrm{CE} \mathrm{CC}$ & $(372)$ \\
\hline Gochnatia pulchra Cabrera & FAN & $\mathrm{CE}$ & $(375,403)$ \\
\hline Gochnatia polymorpha (Less.) Cabrera & FAN & C CE CC & $(396,467)$ \\
\hline Mikania cordifolia (L.f.) Willd. & CAM & CL & $(559)$ \\
\hline Piptocarpha rotundifolia (Less.) Baker & FAN & C CE & $(424,514)$ \\
\hline Pterocaulon alopecuroides (Lam.) DC. & CAM & CS & $(564)$ \\
\hline Pterocaulon angustifolium DC. & CAM & $\mathrm{CCCS}$ & $(525)$ \\
\hline Senecio brasiliensis (Spreng.) Less. & FAN & $\mathrm{CCCS}$ & $(649)$ \\
\hline Senecio aff. oxyphyllus DC. & FAN & $\mathrm{CCCS}$ & $(527)$ \\
\hline Symphyopappus cuneatus (DC.)Sch.Bip. ex Baker & FAN & $\mathrm{CC}$ & $(767)$ \\
\hline Symphyopappus polystachyus Baker & FAN & $\mathrm{CE}$ & (341) \\
\hline Vernonia bardanoides Less & CAM & CS & $(597,768)$ \\
\hline Vernonia chamissonis Less. & FAN & CE CC & $(348,371,510)$ \\
\hline Vernonia cognata Less. & CAM & CS & $(488)$ \\
\hline Vernonia rubriramea Mart. & FAN & $\mathrm{CE}$ & $(366)$ \\
\hline $\begin{array}{l}\text { Vernonia simplex Less. } \\
\text { BEGONIACEAE }\end{array}$ & CAM & CS & $(683,494)$ \\
\hline $\begin{array}{l}\text { Begonia aff. alchemilloides Meisn. ex DC. } \\
\text { BIGNONIACEAE }\end{array}$ & CAM & CL & $(539,562)$ \\
\hline Adenocalymma bracteatum (Cham.) DC. & LIA & $\mathrm{CE}$ & $(382,400)$ \\
\hline Anemopaegma arvense (Vell.) Stellfeld ex Souza & GEO & CE CC CS & $-(516)$ \\
\hline
\end{tabular}

R. Árvore, Viçosa-MG, v.31, n.5, p.907-922, 2007 
Quadro 1 - Cont.

Table 1 - Cont

\begin{tabular}{|c|c|c|c|}
\hline FAMÍLIA/ESPÉCIE & $\begin{array}{l}\text { FORMA } \\
\text { DE VIDA }\end{array}$ & FISIONOMIA & $\begin{array}{l}\text { NÚMERO DE } \\
\text { COLETA }\end{array}$ \\
\hline \multicolumn{4}{|l|}{ BIGNONIACEAE } \\
\hline Anemopaegma aff arvense (Vell.) Stellfeld ex Souza & GEO & $\mathrm{CECC}$ & $(584)$ \\
\hline Arrabidaea chica (Humb. \& Bonpl.) B. Verl. & LIA & $\mathrm{CE}$ & $(457)$ \\
\hline Arrabidaea corallina (Jacq.) Sandwith & LIA & $\mathrm{C}$ & $(598)$ \\
\hline Arrabidaea aff multiflora Bureau \& K. Schum. & LIA & $\mathrm{CE}$ & $(395)$ \\
\hline Jacaranda caroba (Vell.) A. DC. & FAN & $\mathrm{CE}$ & $(393,450,589)$ \\
\hline Jacaranda decurrens Cham. & GEO & CE CC CS & $(693)$ \\
\hline Jacaranda rufa Silva Manso & FAN & $\mathrm{CE}$ & (411) \\
\hline Memora axillaris $\mathrm{K}$. Schum. & FAN & $\mathrm{CE}$ & $(390)$ \\
\hline Pyrostegia venusta (Ker Gawl.) Miers & LIA & $\mathrm{CE}$ & $(392)$ \\
\hline \multicolumn{4}{|l|}{ BIXACEAE } \\
\hline $\begin{array}{l}\text { Cochlospermum regium (Schrank) Pilg. } \\
\text { BORAGINACEAE }\end{array}$ & HEM & $\mathrm{CE}$ & $(703)$ \\
\hline \multicolumn{4}{|l|}{ BROMELIACEAE } \\
\hline Bromelia balansae $\mathrm{Mez}$ & HEM & $\mathrm{C} \mathrm{CE}$ & $(416)$ \\
\hline $\begin{array}{l}\text { Dyckia cf remotiflora Otto \& Dietr. } \\
\text { BURMANNIACEAE }\end{array}$ & HEM & $\mathrm{CC}$ & $(650)$ \\
\hline \multicolumn{4}{|l|}{ CAMPANULACEAE } \\
\hline Lobelia exaltata Pohl & FAN & $\mathrm{CL}$ & $(570)$ \\
\hline $\begin{array}{l}\text { Lobelia nummularioides Cham. } \\
\text { CARYOCARACEAE }\end{array}$ & CAM & no riacho & $(636)$ \\
\hline $\begin{array}{l}\text { Caryocar brasiliense Cambess. } \\
\text { CELASTRACEAE }\end{array}$ & FAN & $\mathrm{C} \mathrm{CE} \mathrm{CC}$ & $(369)$ \\
\hline Austroplenckia populnea (Reissek) Lundell & FAN & $\mathrm{CE}$ & (724) \\
\hline Maytenus aff alaternoides Reissek & FAN & $\mathrm{CE}$ & $(713)$ \\
\hline \multicolumn{4}{|l|}{ CHRYSOBALANACEAE } \\
\hline Couepia grandiflora (Mart. \& Zucc.) Benth. ex Hook. f. & FAN & C CE & (435) \\
\hline \multicolumn{4}{|l|}{ CLUSIACEAE } \\
\hline Kielmeyera coriacea Mart. & FAN & $\mathrm{CE}$ & $(228)$ \\
\hline \multicolumn{4}{|l|}{ COMBRETACEAE } \\
\hline \multicolumn{4}{|l|}{ CONNARACEAE } \\
\hline $\begin{array}{l}\text { Connarus suberosus Planch. } \\
\text { CONVOLVULACEAE }\end{array}$ & FAN & $\mathrm{CECC}$ & $(756)$ \\
\hline Evolvulus filipes Mart. & CAM & CS & $(682)$ \\
\hline Evolvulus aff linoides Moric. & CAM & CS & $(681)$ \\
\hline Iротоеа $\mathrm{sp}$ & LIA & CS & (493) \\
\hline $\begin{array}{l}\text { Merremia macrocalyx (Ruiz \& Pav.) O’Donell } \\
\text { CUCURBITACEAE }\end{array}$ & LIA & $\mathrm{CE}$ & $(761)$ \\
\hline $\begin{array}{l}\text { Cayaponia ternata } \operatorname{Cog} \mathrm{n} . \\
\text { CYPERACEAE }\end{array}$ & LIA & $\mathrm{CE}$ & $(507)$ \\
\hline Bulbostylis capillaris (L.) C.B.Clarke & HEM & CL & $(567)$ \\
\hline Rhynchospora cf albiceps Kunth & HEM & CL & $(689,626)$ \\
\hline
\end{tabular}


Quadro 1-Cont.

Table 1 -Cont.

\begin{tabular}{|c|c|c|c|}
\hline FAMÍLIA/ESPÉCIE & $\begin{array}{l}\text { FORMA } \\
\text { DE VIDA }\end{array}$ & FISIONOMIA & $\begin{array}{l}\text { NÚMERO DE } \\
\text { COLETA }\end{array}$ \\
\hline \multicolumn{4}{|l|}{ CYPERACEAE } \\
\hline Rhynchospora consanguinea (Kunth) Boeck. & HEM & CL & $(627)$ \\
\hline Rhynchospora exaltata Kunth & HEM & $\mathrm{C} \mathrm{CE}$ & (353) \\
\hline Rhynchospora lundii Boeck & HEM & CL & $(566)$ \\
\hline $\begin{array}{l}\text { Rhynchospora marisculus Lindl. ex Nees } \\
\text { DILLENIACEAE }\end{array}$ & HEM & CL & $(569)$ \\
\hline $\begin{array}{l}\text { Davilla rugosa Poir. } \\
\text { DROSERACEAE }\end{array}$ & FAN & $\mathrm{CE}$ & $(352)$ \\
\hline $\begin{array}{l}\text { Drosera intermedia Hayne } \\
\text { EBENACEAE }\end{array}$ & HEM & CL & $(760)$ \\
\hline $\begin{array}{l}\text { Diospyros hispida A. DC. } \\
\text { ERICACEAE }\end{array}$ & FAN & $\mathrm{CE}$ CC & $(663)$ \\
\hline Gaylussacia brasiliensis (Spreng.) Meisn. & FAN & M & $(618)$ \\
\hline Gaylussacia pseudogaultheria Cham. \& Schltdl. & FAN & M & $(640)$ \\
\hline $\begin{array}{l}\text { Leucothoe serrulata (Cham.) DC. } \\
\text { ERIOCAULACEAE }\end{array}$ & FAN & M & $(646)$ \\
\hline Paepalanthus macrotrichus Silveira & TER & CL & $(535)$ \\
\hline Syngonanthus caulescens (Poir.) Ruhland & TER & CL & $(635)$ \\
\hline Syngonanthus gracilis (Bong.) Ruhland & TER & CL & $(603,624)$ \\
\hline Syngonanthus helminthorrhizus (Mart.) Ruhland & TER & CL & $(622,533,692)$ \\
\hline Syngonanthus nitens (Bong.) Ruhland & TER & CL & (691) \\
\hline $\begin{array}{l}\text { Syngonanthus xeranthemoides (Bong.) Ruhland } \\
\text { ERYTHROXYLACEAE }\end{array}$ & TER & CL & $(688)$ \\
\hline Erythroxylum campestre A. St.-Hil. & FAN & CE CC CS & $(668,563,491)$ \\
\hline Erythroxylum cuneifolium (Mart.) O. E. Schulz & FAN & C CE CC & $(409,460,639)$ \\
\hline Erythroxylum suberosum A. St.-Hil. & FAN & C CE CC & $(658)$ \\
\hline $\begin{array}{l}\text { Erythroxylum tortuosum Mart. } \\
\text { EUPHORBIACEAE }\end{array}$ & FAN & $\mathrm{CE}$ & $(721)$ \\
\hline Actinostemon communis (Müll. Arg.) Pax & FAN & $\mathrm{C} \mathrm{M}$ & $(608)$ \\
\hline Manihot tripartita (Spreng.) Müll. Arg. & FAN & CE & $(551)$ \\
\hline Pera glabrata (Schott) Poepp. ex Baill. & FAN & C CE M & (723) \\
\hline Sapium biglandulosum (L.) Müll. Arg. & FAN & M & $(647)$ \\
\hline Sapium sp & FAN & $\mathrm{CE}$ & (342) \\
\hline $\begin{array}{l}\text { Sebastiania serrulata (Mart.) Müll. Arg. } \\
\text { FABACEAE CAESALPINIOIDEAE }\end{array}$ & CAM & $\mathrm{CE}$ & $(464,764)$ \\
\hline Bauhinia bongardii Steud. & FAN & $\mathrm{CE}$ & $(585)$ \\
\hline Bauhinia rufa (Bong.) Steud. & FAN & C CE CC & (397) \\
\hline Cassia bicapsularis $\mathrm{L}$. & FAN & M & $(547)$ \\
\hline Cassia flexиosa $\mathrm{L}$. & CAM & $\mathrm{CE} C \mathrm{C}$ & $(351,540)$ \\
\hline Cassia rotundifolia Pers. & CAM & $\mathrm{CS}$ & $(652)$ \\
\hline Cassia rugosa G. Don & FAN & $\mathrm{CE}$ & $(387,412,511)$ \\
\hline Cassia splendida Vogel & LIA & $\mathrm{CE}$ & $(386)$ \\
\hline Cassia sp. & CAM & $\mathrm{CE}$ & $(427)$ \\
\hline Copaifera langsdorffii Desf. & FAN & C CE & $(750)$ \\
\hline Dimorphandra mollis Benth. & FAN & $\mathrm{C} \mathrm{CE} \mathrm{CC}$ & $(418,508)$ \\
\hline $\begin{array}{l}\text { Hymenaea stigonocarpa Mart. ex Hayne } \\
\text { FABACEAE FABOIDEAE }\end{array}$ & FAN & $\mathrm{CE}$ & $(725)$ \\
\hline Acosmium subelegans (Mohlenbr.) Yakovlev & FAN & C CE CC & $(479,726)$ \\
\hline Andira laurifolia Benth. & GEO & CE CC CS & $(523,653)$ \\
\hline
\end{tabular}


Quadro 1 - Cont

Table 1 - Cont.

\begin{tabular}{|c|c|c|c|}
\hline FAMÍLIA/ESPÉCIE & $\begin{array}{l}\text { FORMA } \\
\text { DE VIDA }\end{array}$ & FISIONOMIA & $\begin{array}{l}\text { NÚMERO DE } \\
\text { COLETA }\end{array}$ \\
\hline \multicolumn{4}{|l|}{ FABACEAE FABOIDEAE } \\
\hline Bowdichia virgilioides Kunth & FAN & $\mathrm{CE}$ & $(751)$ \\
\hline Camptosema ellipticum (Desv.) Burkart & LIA & M & (699) \\
\hline Centrosema bracteosum Benth. & LIA & $\mathrm{CE}$ & (483) \\
\hline Crotalaria micans Link & CAM & CS & $(660)$ \\
\hline Dalbergia miscolobium Benth. & FAN & $\mathrm{CE}$ & (755) \\
\hline Eriosema aff congestum Benth. & CAM & CS & $(662)$ \\
\hline Eriosema heterophyllum Benth. & CAM & $\mathrm{CC}$ & $(623)$ \\
\hline Machaerium acutifolium Vogel & FAN & C CE CC & $(350,474)$ \\
\hline Rhynchosia minima (L.) DC. & LIA & $\mathrm{C}$ & (556) \\
\hline Stylosanthes scabra Vogel & HEM & CS & $(746)$ \\
\hline \multicolumn{4}{|l|}{ FABACEAE MIMOSOIDEAE } \\
\hline Anadenanthera falcata (Benth.) Speg. & FAN & $\mathrm{C} \mathrm{CE}$ & $(753)$ \\
\hline Calliandra foliolosa Benth. & FAN & M & $(700)$ \\
\hline Mimosa acerba Benth. & CAM & $\mathrm{CE}$ CC & $(364,519)$ \\
\hline Mimosa rixosa Mart. & CAM & $\mathrm{CCCS}$ & $(530)$ \\
\hline Pithecellobium incuriale (Vell.) Benth. & FAN & $\mathrm{C}$ & $(755)$ \\
\hline Plathymenia reticulata Benth. & FAN & $\mathrm{C} \mathrm{CE}$ & $(708)$ \\
\hline Stryphnodendron adstringens (Mart.) Coville & FAN & $\mathrm{CE}$ & $(373)$ \\
\hline $\begin{array}{l}\text { Stryphnodendron polyphyllum Mart. } \\
\text { GENTIANACEAE }\end{array}$ & FAN & C CE CC & $(463,370)$ \\
\hline Lisianthus aff. chelonoides L. f. & CAM & CL & $(531)$ \\
\hline \multicolumn{3}{|l|}{ JUNCACEAE } & $(385)$ \\
\hline Juncus scirpoides Lam. & HEM & CL & $(686)$ \\
\hline \multicolumn{3}{|l|}{ LACISTEMATACEAE } & (628) \\
\hline \multicolumn{4}{|l|}{ LAMIACEAE } \\
\hline Eriope crassipes Benth. & CAM & $\mathrm{CE}$ & $(695)$ \\
\hline Hyptis balansae Briq. & CAM & $\mathrm{CE}$ & $(382,444,424)$ \\
\hline Hyptis lacustris A. St.-Hil. ex Benth. & CAM & CL & $(685)$ \\
\hline \multicolumn{3}{|l|}{ LAURACEAE } & $(674)$ \\
\hline Ocotea brasiliensis Coe-Teix. & FAN & M & (548) \\
\hline Ocotea corymbosa (Meisn.) Mez & FAN & C CE CC & (367) \\
\hline Ocotea pulchella Mart. & FAN & C CE & $(347,471)$ \\
\hline Persea pyrifolia Nees & FAN & $\mathrm{C}$ & $(454)$ \\
\hline \multicolumn{4}{|l|}{ LENTIBULARIACEAE } \\
\hline Utricularia cuculata A.St.-Hil. \& Girard & HEM & CL & (534) \\
\hline Utricularia nana A. St.-Hil. \& Girard & TER & CL & $(393,583)$ \\
\hline $\begin{array}{l}\text { Utricularia nervosa } \text { G. Weber ex Benj. } \\
\text { LOGANIACEAE }\end{array}$ & TER & $\mathrm{CL}$ & $(592,631)$ \\
\hline $\begin{array}{l}\text { Strychnos brasiliensis (Spreng.) Mart. } \\
\text { LORANTHACEAE }\end{array}$ & FAN & M & $(704)$ \\
\hline $\begin{array}{l}\text { Struthanthus aff complexus Eichler } \\
\text { LYTHRACEAE }\end{array}$ & EPI & $\mathrm{CE}$ & $(429)$ \\
\hline $\begin{array}{l}\text { Lafoensia replicata Pohl } \\
\text { MALPIGHIACEAE }\end{array}$ & FAN & $\mathrm{C} \mathrm{CE}$ & $(707)$ \\
\hline Banisteriopsis campestris (A. Juss.) Little & FAN & - CE & $(475)$ \\
\hline
\end{tabular}

R. Árvore, Viçosa-MG, v.31, n.5, p.907-922, 2007 
Quadro 1 - Cont.

Table 1 -Cont.

\begin{tabular}{|c|c|c|c|}
\hline FAMÍLIA/ESPÉCIE & $\begin{array}{c}\text { FORMA } \\
\text { DE VIDA }\end{array}$ & FISIONOMIA & $\begin{array}{l}\text { NÚMERO DE } \\
\text { COLETA }\end{array}$ \\
\hline \multicolumn{4}{|l|}{ MALPIGHIACEAE } \\
\hline Banisteriopsis laevifolia (A. Juss.) B. Gates & FAN & $\mathrm{CC}$ & $(543)$ \\
\hline Banisteriopsis malifolia (Nees \& Mart.) B. Gates & LIA & $\mathrm{CE}$ & $(408,588)$ \\
\hline Banisteriopsis stellaris (Griseb.) B. Gates & LIA & $\mathrm{CE}$ & $(360,365)$ \\
\hline Banisteriopsis variabilis $\mathrm{B}$. Gates & LIA & $\mathrm{CE}$ & $(361)$ \\
\hline Byrsonima coccolobaefolia Kunth & FAN & $\mathrm{CE}$ & $(711)$ \\
\hline Byrsonima intermedia A. Juss. & FAN & C CE CC CS & $(399,274,679)$ \\
\hline Byrsonima subterranea Brade \& Markgr. & GEO & CS & $(485)$ \\
\hline Heteropteris campestris A. Juss. & LIA & $\mathrm{C}$ & $(590)$ \\
\hline Heteropteris coriacea A. Juss. & FAN & M & $(637)$ \\
\hline Heteropteris $\mathrm{sp}$ & FAN & $\mathrm{CE}$ & $(730)$ \\
\hline Mascagnia cordifolia (A. Juss.) Griseb. & FAN & $\mathrm{CE}$ & (469) \\
\hline Peixotoa reticulata Griseb. & FAN & $\mathrm{CE}$ & $(553)$ \\
\hline \multicolumn{4}{|l|}{ MALVACEAE } \\
\hline Byttneria oblongata Pohl & CAM & CS & $(500,656)$ \\
\hline Eriotheca gracilipes (K.Schum.) A.Robyns & FAN & $\mathrm{CE} \mathrm{CC}$ & $(382,407)$ \\
\hline Helicteres brevispira A. St.-Hil. & FAN & M & $(610)$ \\
\hline Luehea grandiflora Mart. \& Zucc. & FAN & $\mathrm{CE}$ & $(398,426)$ \\
\hline Pavonia malacophylla (Link \& Otto) Garcke & HEM & $\mathrm{CCCS}$ & $(481,544)$ \\
\hline Waltheria communis A. St.-Hil. & CAM & CS & $(651)$ \\
\hline \multicolumn{4}{|l|}{ MAYACACEAE } \\
\hline Mayaca sellowiana Kunth & HEM & CL & $(634)$ \\
\hline \multicolumn{4}{|l|}{ MELASTOMATACEAE } \\
\hline Acisanthera alsinaefolia (DC.) Triana & CAM & $\mathrm{CL}$ & $(596,693)$ \\
\hline Acisanthera variabilis (Mart.) Triana & CAM & $\mathrm{CL}$ & $(576)$ \\
\hline Leandra cf xanthopogon Cogn. & FAN & $\mathrm{CE}$ & $(391)$ \\
\hline Miconia albicans (Sw.) Triana & FAN & C CE CC & (423) \\
\hline Miconia chamissois Naudin & FAN & M & $(560,621)$ \\
\hline Miconia ligustroides (DC.) Naudin & FAN & $\mathrm{C} \mathrm{CE}$ & $(379,461,509,709)$ \\
\hline Miconia langsdorffii Cogn. & FAN & $\mathrm{C} \mathrm{CE}$ & $(362,523)$ \\
\hline Miconia sellowiana Naudin & FAN & $\mathrm{CE}$ & $(710)$ \\
\hline Miconia fallax DC. & FAN & C CE CC & $(422,437,612)$ \\
\hline Miconia theaezans (Bonpl.) Cogn. & FAN & $\mathrm{CL}$ & $(581)$ \\
\hline Microlepis oleaefolia (DC.) Triana & FAN & $\mathrm{CL}$ & $(561,763)$ \\
\hline Microlicia humilis Naudin & CAM & $\mathrm{CL}$ & $(529,601)$ \\
\hline Pterolepis longistyla Cogn. & CAM & $\mathrm{CL}$ & $(537,577,594)$ \\
\hline Rhynchanthera hispida Naudin & FAN & $\mathrm{CL}$ & $(762)$ \\
\hline \multicolumn{3}{|l|}{ MELIACEAE } & $(528,595,602)$ \\
\hline Cedrela odorata $\mathrm{L}$. & FAN & M & $(666)$ \\
\hline Guarea macrophylla Vahl & FAN & M & $(645)$ \\
\hline \multicolumn{4}{|l|}{ MONIMIACEAE } \\
\hline \multicolumn{4}{|l|}{ MORACEAE } \\
\hline Brosimum gaudichaudii Trécul & FAN & $\mathrm{CE}$ & $(611)$ \\
\hline \multicolumn{4}{|l|}{ MYRISTICACEAE } \\
\hline Virola gardneri (A.DC.) Warb. & FAN & $\mathrm{C} \mathrm{CE}$ & $(766)$ \\
\hline \multicolumn{4}{|l|}{ MYRSINACEAE } \\
\hline Rapanea ferruginea (Ruiz \& Pav.) Mez & FAN & $\mathrm{C} \mathrm{CE}$ & $(384)$ \\
\hline
\end{tabular}

R. Árvore, Viçosa-MG, v.31, n.5, p.907-922, 2007 
Quadro 1 - Cont

Table 1 - Cont

\begin{tabular}{|c|c|c|c|}
\hline FAMÍLIA/ESPÉCIE & $\begin{array}{l}\text { FORMA } \\
\text { DE VIDA }\end{array}$ & FISIONOMIA & $\begin{array}{l}\text { NÚMERO DE } \\
\text { COLETA }\end{array}$ \\
\hline \multicolumn{4}{|l|}{ MYRSINACEAE } \\
\hline Rapanea guianensis Aubl. & FAN & $\mathrm{CE}$ & (712) \\
\hline Rapanea lancifolia $\mathrm{Mez}$ & FAN & C CE & $(345,462)$ \\
\hline $\begin{array}{l}\text { Rapanea umbellata (Mart.) Mez } \\
\text { MYRTACEAE }\end{array}$ & FAN & C CE & $(402)$ \\
\hline Campomanesia adamantium (Cambess.) O. Berg & FAN & C CE CC CS & $(368,659,665)$ \\
\hline Campomanesia xanthocarpa O. Berg & FAN & M & (702) \\
\hline Eugenia aurata $\mathrm{O}$. Berg & FAN & CE CC CS & $(671,654,743)$ \\
\hline Eugenia bimarginata DC. & FAN & CE CC CS & $(426,599,586)$ \\
\hline Eugenia cf mansoni O. Berg & FAN & $\mathrm{CE}$ & $(378,446)$ \\
\hline Eugenia aff oblongata Mattos \& D. Legrand & FAN & $\mathrm{CE}$ & $(742)$ \\
\hline Eugenia springiana $\mathrm{O}$. Berg & CAM & CE & $(440)$ \\
\hline Eugenia uniflora L. & FAN & $\mathrm{CE}$ & $(447)$ \\
\hline Eugenia aff uniflora $\mathrm{L}$. & FAN & $\mathrm{CE}$ & $(617)$ \\
\hline Myrceugenia aff alpigena (DC.) Landrum & FAN & $\mathrm{CC}$ & $(677)$ \\
\hline Myrceugenia glaucescens (Cambess.) D. Legrand \& Kausel & CAM & $\mathrm{CE}$ & $(428)$ \\
\hline Myrcia laruotteana Cambess. & FAN & $\mathrm{C} \mathrm{CE}$ & $(430,431,607)$ \\
\hline Myrcia lasiantha DC. & FAN & C CE CC & $(466,517)$ \\
\hline Myrcia lingua (O. Berg) Mattos \& D. Legrand & FAN & C CE CC CS & $(671,654)$ \\
\hline Myrcia multiflora (Lam.) DC. & FAN & $\mathrm{C} \mathrm{CE}$ & $(453)$ \\
\hline Myrcia tomentosa (Aubl.) DC. & FAN & C CE CC & $(505,733)$ \\
\hline Myrcia sp. & FAN & $\mathrm{C}$ & $(732)$ \\
\hline Myrciaria sp & CAM & $\mathrm{CE}$ & $(441,448)$ \\
\hline Psidium aff australe Cambess & FAN & $\mathrm{CE}$ & $(455)$ \\
\hline \multicolumn{4}{|l|}{ NYCTAGINACEAE } \\
\hline Guapira noxia (Netto) Lundell & FAN & $\mathrm{C} \mathrm{CE}$ & (738) \\
\hline $\begin{array}{l}\text { Guapira opposita (Vell.) Reitz } \\
\text { OCHNACEAE }\end{array}$ & \multicolumn{2}{|c|}{ OCHNACEAE } & $(739)$ \\
\hline Ouratea spectabilis (Mart. ex Eng1.) Engl. & FAN & C CE CC & (443) \\
\hline $\begin{array}{l}\text { Sauvagesia sp. } \\
\text { ONAGRACEAE }\end{array}$ & CAM & CL & $(578)$ \\
\hline $\begin{array}{l}\text { Ludwigia nervosa (Poir.) H. Hara } \\
\text { OPILIACEAE }\end{array}$ & FAN & $\mathrm{CL}$ & $(767)$ \\
\hline $\begin{array}{l}\text { Agonandra brasiliensis Miers ex Benth. \& Hook. f. } \\
\text { ORCHIDACEAE }\end{array}$ & FAN & $\mathrm{CE}$ & $(731)$ \\
\hline Epidendrum ellipticum Graham & HEM & $\mathrm{CE}$ & $(388)$ \\
\hline Epistephium sclerophyllum Lindl. & HEM & $\mathrm{CC}$ & $(542)$ \\
\hline $\begin{array}{l}\text { Rodriguezia aff rigida (Lindl.) Rchb. f. } \\
\text { PIPERACEAE }\end{array}$ & HEM & $\mathrm{CE}$ & $(394)$ \\
\hline $\begin{array}{l}\text { Peperomia glabella (Sw.) A. Dietr. } \\
\text { POACEAE }\end{array}$ & CAM & M & $(642)$ \\
\hline Andropogon bicornis L. & HEM & CL & $(568,768)$ \\
\hline Andropogon leucostachyus Kunth & HEM & CL & $(638)$ \\
\hline Aristida jubata (Arechav.) Herter & HEM & CE CC CS & $(670)$ \\
\hline Aristida riparia Trin. & HEM & CE CC CS & $(573)$ \\
\hline Axonopus brasiliensis (Spreng.) Kuhlm. & HEM & $\mathrm{CL}$ & $(605)$ \\
\hline Axonopus pressus (Nees ex Steud.) Parodi & HEM & CE CC CS & $(574)$ \\
\hline Eragrostis maypurensis (Kunth) Steud. & HEM & $\mathrm{CL}$ & $(604)$ \\
\hline Eragrostis sp. ----------- & HEM & - CE CC CS & $(571)$ \\
\hline
\end{tabular}

R. Árvore, Viçosa-MG, v.31, n.5, p.907-922, 2007 
Quadro 1-Cont.

Table 1 -Cont.

\begin{tabular}{|c|c|c|c|}
\hline FAMÍLIA/ESPÉCIE & $\begin{array}{l}\text { FORMA } \\
\text { DE VIDA }\end{array}$ & FISIONOMIA & $\begin{array}{l}\text { NÚMERO DE } \\
\text { COLETA }\end{array}$ \\
\hline \multicolumn{4}{|l|}{ POACEAE } \\
\hline Gymnopogon foliosus (Willd.) Nees & HEM & CE CC CS & $(572,358)$ \\
\hline Olyra micrantha Kunth & HEM & $\mathrm{CE}$ & $(451)$ \\
\hline Panicum olyroides Kunth & HEM & CE CC CS & $(664)$ \\
\hline Rhynchelytrum repens (Willd.) C.E.Hubb. & HEM & $\mathrm{CE}$ & (747) \\
\hline Sporobolus sp. & HEM & CE CC CS & $(667)$ \\
\hline \multicolumn{3}{|l|}{ POLYGALACEAE } & $(673)$ \\
\hline Bredemeyera floribunda Willd. & LIA & $\mathrm{C}$ & $(552)$ \\
\hline Polygala fimbriata A.W. Benn. & CAM & M & $(521)$ \\
\hline Polygala sabulosa A.W. Benn. & CAM & CL & $(632)$ \\
\hline Polygala tenuis DC. & CAM & CL & $(592,633)$ \\
\hline \multicolumn{4}{|l|}{ POLYGONACEAE } \\
\hline \multicolumn{4}{|l|}{ PROTEACEAE } \\
\hline \multicolumn{4}{|l|}{ RHAMNACEAE } \\
\hline Crumenaria polygaloides Reissek & CAM & CE CC CS & $(675,698)$ \\
\hline Frangula polymorpha Reissek & FAN & CE CL M & (558) \\
\hline \multicolumn{3}{|l|}{ ROSACEAE } & $(550)$ \\
\hline \multicolumn{4}{|l|}{ RUBIACEAE } \\
\hline Alibertia sessilis (Vell.) K. Schum. & FAN & C CE & (741) \\
\hline Amaioua guianensis Aubl. & FAN & $\mathrm{C}$ & (383) \\
\hline Borreria poaya (A. St.-Hil) DC. & CAM & CS & $(486)$ \\
\hline Coccocypselum canescens Willd. ex Schult. \& Schult. f. & CAM & $\mathrm{C} \mathrm{CE}$ & $(380)$ \\
\hline Declieuxia chiococcoides Humb., Bonpl. \& Kunth & CAM & $\mathrm{CE}$ & $(470)$ \\
\hline Guettarda viburnoides Cham. \& Schltdl. & FAN & M & (413) \\
\hline Manettia cordifolia Mart. & LIA & M & $(546)$ \\
\hline Palicourea nicotianaefolçia Cham. \& Schltdl. & CAM & CS & (496) \\
\hline Psychotria barbiflora DC. & CAM & $\mathrm{C}$ & (363) \\
\hline Psychotria sessilis Vell. & FAN & C CE & $(414,456)$ \\
\hline Relbunium buxifolium K. Schum. & CAM & M & (641) \\
\hline Sipanea pratensis Aubl. & CAM & CL & $(536)$ \\
\hline Tocoyena formosa (Cham. \& Schltdl.) K. Schum. & FAN & C CE & $(472,520)$ \\
\hline $\begin{array}{l}\text { Ixora gardneriana Benth. } \\
\text { RUTACEAE }\end{array}$ & FAN & $\mathrm{CE}$ & (729) \\
\hline $\begin{array}{l}\text { Zanthoxylum rhoifolium Lam. } \\
\text { SALICACEAE }\end{array}$ & FAN & $\mathrm{C} \mathrm{CE}$ & $(735)$ \\
\hline Casearia lasiophylla Eichler & FAN & CE M & $(349,445)$ \\
\hline $\begin{array}{l}\text { Casearia sylvestris } \mathrm{Sw} . \\
\text { SAPINDACEAE }\end{array}$ & FAN & $\mathrm{C} \mathrm{CE}$ & $(420,476,620)$ \\
\hline Allophylus edulis (A. St.-Hil., Cambess. \& A. Juss.) Radlk. & FAN & CE M & (736) \\
\hline Matayba elaeagnoides Radlk. & FAN & CE M & (737) \\
\hline $\begin{array}{l}\text { Serjania lethalis A. St.-Hil. } \\
\text { SAPOTACEAE }\end{array}$ & LIA & $\mathrm{CE}$ & (419) \\
\hline Chrysophyllum marginatum (Hook. \& Arn.) Radlk. & FAN & CE M & (745) \\
\hline Pouteria ramiflora (Mart.) Radlk. & FAN & $\mathrm{C}$ & $(452)$ \\
\hline
\end{tabular}

R. Árvore, Viçosa-MG, v.31, n.5, p.907-922, 2007 
Quadro 1 - Cont

Table 1 - Cont.

\begin{tabular}{|c|c|c|c|}
\hline FAMÍLIA/ESPÉCIE & $\begin{array}{l}\text { FORMA } \\
\text { DE VIDA }\end{array}$ & FISIONOMIA & $\begin{array}{l}\text { NÚMERO DE } \\
\text { COLETA }\end{array}$ \\
\hline \multicolumn{4}{|l|}{ SCROPHULARIACEAE } \\
\hline Buddleja brasiliensis Jacq. ex Spreng. & CAM & M & $(606)$ \\
\hline Esterhazya splendida J.C. Mikan & FAN & CL & $(579)$ \\
\hline SMILACACEAE & & & \\
\hline $\begin{array}{l}\text { Smilax aff robusta Griseb. } \\
\text { SOLANACEAE }\end{array}$ & LIA & $\mathrm{C} \mathrm{CE}$ & $(404)$ \\
\hline Cestrum sendtnerianum Mart. ex Sendtn. & FAN & $\mathrm{CE}$ & $(438)$ \\
\hline $\begin{array}{l}\text { Solanum lycocarpum A. St.-Hil. } \\
\text { STYRACACEAE }\end{array}$ & FAN & $\mathrm{CC} \mathrm{CE}$ & $(503)$ \\
\hline Styrax camporum Pohl & FAN & $\mathrm{C} \mathrm{CE}$ & (343) \\
\hline $\begin{array}{l}\text { Styrax ferrugineus Nees \& Mart. } \\
\text { SYMPLOCACEAE }\end{array}$ & FAN & C CE CC & $(344)$ \\
\hline $\begin{array}{l}\text { Symplocos pubescens Klotzsch ex Benth. } \\
\text { THYMELAEACEAE }\end{array}$ & FAN & $\mathrm{C} \mathrm{CE}$ & $(346,425)$ \\
\hline $\begin{array}{l}\text { Daphnopsis fasciculata (Meisn.) Nevling } \\
\text { VERBENACEAE }\end{array}$ & FAN & $\mathrm{C} \mathrm{CE}$ & $(734)$ \\
\hline Aegiphila sellowiana Cham. & FAN & $\mathrm{CECC}$ & $(374,406,696)$ \\
\hline Lantana camara $\mathrm{L}$ & FAN & $\mathrm{CE}$ & $(442)$ \\
\hline Lyppia corymbosa Cham. & FAN & C CE CC & $(512)$ \\
\hline $\begin{array}{l}\text { Lyppia lupulina Cham. } \\
\text { VOCHYSIACEAE }\end{array}$ & CAM & CE CC CS & $(432,672)$ \\
\hline Qualea multiflora Mart. & FAN & $\mathrm{CE}$ & $(600)$ \\
\hline Vochysia cinnamomea Pohl & FAN & $\mathrm{CE}$ & $(769)$ \\
\hline $\begin{array}{l}\text { Vochysia tucanorum Mart. } \\
\text { XYRIDACEAE }\end{array}$ & FAN & C CE M & $(744)$ \\
\hline Xyris capensis Thunb. & TER & $\mathrm{CL}$ & $(687)$ \\
\hline Xyris jupicai Rich. & TER & CL & $(690)$ \\
\hline Xyris aff regnellii L.A.Nilsson & HEM & $\mathrm{CL}$ & $(582)$ \\
\hline Xyris tortula Mart. & HEM & CL & $(532,643)$ \\
\hline
\end{tabular}

Se considerar as estimativas de riqueza feita por Castro et al. (1999), admite-se um número entre 3.000 e 7.000 espécies nos 200 milhões de hectares da distribuição original do Cerrado. Ocupando uma área correspondente a $0,0014 \%$ da área original de Cerrado, a EESB possuiria de 4 a 9,5\% da totalidade de espécies de Cerrado.

As famílias com maior número de espécies coletadas na EESB, incluindo as Matas de Galeria, foram Asteraceae (33 espécies), Fabaceae (31, sendo 11 Caesalpinioideae, 12 Faboideae e 8 Mimosoideae), Myrtaceae (20), Melastomataceae (15), Poaceae (14), Rubiaceae (14), Malpighiaceae (13) e Bignoniaceae (12). Os gêneros mais ricos foram Miconia (Melastomataceae), Eupatorium (Asteraceae) e Eugenia (Myrtaceae), com sete espécies; Myrcia (Myrtaceae) e Cassia (Caesalpiniaceae), com seis espécies.
No Cerrado sensu lato, que na EESB foi o conjunto formado por campo limpo úmido, campo sujo, campo cerrado, Cerrado sensu stricto e Cerradão, as famílias mais ricas foram Asteraceae (32 espécies), Fabaceae (28, sendo 10 Caesalpinioideae, 11 Faboideae e 7 Mimosoideae), Myrtaceae (19), Poaceae (14), Malpighiaceae (13), Bignoniaceae (12) e Rubiaceae (11).

Houve diferenças no número de espécies entre as diferentes fitofisionomias de Cerrado. Foi encontrado maior número de espécies nas fitofisionomias de Cerrado sensu stricto e no Cerradão, diferindo do observado na Reserva Biológica de Moji-Guaçu e na Reserva Péde-Gigante, em Santa Rita do Passa Quatro, ambas situadas no Estado de São Paulo, onde foram encontradas mais espécies nas fitofisionomias campestres que no Cerrado sensu stricto e no Cerradão (MANTOVANI e MARTINS, 1993; BATALHA e MANTOVANI, 2001). 
Portanto, é possível que mais espécies sejam encontradas nas fitofisionomias de campo cerrado, campo sujo e campo limpo da EESB, além das relacionadas neste estudo.

Mantovani e Martins (1993) e Batalha e Mantovani (2001), ao considerarem a distribuição das espécies nos componentes arbustivo-arbóreo e herbáceosubarbustivo da vegetação de Cerrado da Reserva Biológica de Moji-Guaçu e na Reserva Pé-de-Gigante, respectivamente, encontraram números de espécies aproximados aos obtidos na EESB de Asteraceae (Compositae) e Leguminosae, as duas famílias mais ricas nesses três estudos. A família Asteraceae foi a mais rica tanto em Águas de Santa Bárbara quanto em Moji-Guaçu, ficando as leguminosas como a segunda família mais diversa, o inverso sendo observado na Reserva Pé-de-Gigante. Mendonça et al. (1998), numa compilação de vários trabalhos florísticos desenvolvidos em diferentes vegetações no Domínio de Cerrado, concluíram que Leguminosae, Compositae (Asteraceae), Orchidaceae e Gramineae (Poaceae), em ordem decrescente, são as famílias mais ricas. Batalha e Martins $(2002,2004)$ encontraram riqueza equivalente entre Asteraceae e Leguminosae nas espécies levantadas no Cerrado do Parque Nacional das Emas, em Goiás, seguidas de Poaceae, Myrtaceae e Lamiaceae. Os resultados encontrados na EESB corroboram um padrão florístico que vem sendo evidenciado na vegetação de Cerrado sensu lato como um todo. Assim, as famílias mais ricas do Cerrado sensu lato nos levantamentos completos realizados são Asteraceae e Leguminosae. Essas duas famílias são as mais ricas do Cerrado em todos os levantamentos que estudaram as floras lenhosa e herbáceo-subarbustiva e estão bem-representadas nessas duas floras. Figueiras (2002) citou a família Poaceae como a predominante no estrato herbáceo do Cerrado, sendo incluída entre as mais ricas em número de espécies, e sua riqueza aumenta quando existem maiores proporções de áreas campestres, pois são representadas exclusivamente na flora herbáceosubarbustiva. Talvez por isso as gramíneas não sejam tão ricas quanto as compostas e leguminosas no Cerrado, que têm numerosas espécies lenhosas e herbáceosubarbustivas. As compostas se apresentam com maior número de espécies de menor porte, ocorrendo o inverso com as leguminosas. Em termos de riqueza, famílias como Myrtaceae, Malpighiaceae, Orchidaceae, Lamiaceae e Rubiaceae também estão incluídas entre as mais diversas, mas não têm a constância de compostas, leguminosas e gramíneas, sempre apontadas entre as mais ricas em todos os estudos. Futuros levantamentos conjuntos de flora lenhosa e de flora herbáceo-subarbustiva mostrarão quanto esse padrão é generalizado no domínio de Cerrado.

O espectro biológico de espécies para o Cerrado sensu lato foi de 149 fanerófitas $(51,20 \%), 61$ caméfitas $(20,96 \%), 41$ hemicriptófitas $(14,09 \%)$, seis geófitas (2,06\%), 10 terófitas (3,44\%), 23 lianas (7,90\%) e uma semi-parasita $(0,34 \%)$ (Tabela 1 e Figura 2). No Cerradão, o espectro biológico apresentou 64 fanerófitas $(85,33 \%)$, uma caméfita $(1,33 \%)$, três hemicriptófitas $(4,00 \%)$ e sete lianas $(9,33 \%)$. No Cerrado sensu stricto foram encontradas 120 fanerófitas $(67,41 \%), 17$ caméfitas $(9,55 \%), 19$ hemicriptófitas $(10,67 \%)$, cinco geófitas $(2,81 \%), 16$ lianas $(8,99 \%)$ e uma semiparasita $(0,56 \%)$. No campo Cerrado, o espectro biológico mostrou 47 fanerófitas $(60,26 \%), 11$ caméfitas $(14,10 \%), 15$ hemicriptófitas $(19,23 \%)$ e cinco geófitas $(6,41 \%)$. No campo sujo foram encontradas 11 fanerófitas $(17,46 \%)$, 26 caméfitas $(41,27 \%), 20$ hemicriptófitas $(31,75 \%)$, cinco geófitas $(7,94 \%)$ e uma liana $(1,59 \%)$. O espectro biológico do campo limpo apresentou sete fanerófitas (14\%), 17 caméfitas (34\%), 16 hemicriptófitas (32\%) e 10 terófitas (5\%) (Tabela 1 e Figura 2).

Os espectros biológicos (Figura 2) mostraram tendência de aumento na proporção de espécies das formas de vida fanerofítica e das lianas quando se analisou a seqüência fitofisionômica no sentido do campo limpo para o Cerradão e Mata de Galeria. No mesmo sentido fitofisionômico, observou-se o decréscimo da proporção de espécies das formas de vida camefítica, hemicriptofítica e terofítica. Conforme foi verificado, é esperado que haja aumento na proporção de espécies fanerófitas ao longo do gradiente fisionômico, no sentido das formas savânicas mais abertas para as mais fechadas no Cerrado (COUTINHO, 1978; BATALHA e MARTINS, 2004). Embora fosse esperado que as hemicriptófitas prevalecessem nas formas savânicas mais abertas de Cerrado (BATALHA e MARTINS, 2002), na EESB prevaleceram as caméfitas no campo limpo e no campo sujo. Essa diferença pode ter sido causada por fatores ambientais que não foram investigados. Entre uma infinidade de fatores ambientais, o fogo é sempre importante em ecologia de savanas. A passagem do fogo foi constatada como fator ambiental beneficiador da forma de vida hemicriptofítica em detrimento da camefítica e fanerofítica em espectros biológicos específicos, conforme demonstrado por Meira Neto et al. (2005) em vegetação de fitofisionomia savânica

R. Árvore, Viçosa-MG, v.31, n.5, p.907-922, 2007 
de Muçunungas. Como efeito da ocorrência de incêndios na riqueza, Moreira (2000) relatou a ausência de cinco entre 10 das mais importantes espécies lenhosas de Cerradão em locais não-protegidos do fogo no Jardim Botânico de Brasília. O longo histórico de proteção contra incêndios da EESB pode ter influenciado, positivamente, as caméfitas e fanerófitas, o que se evidencia nas fisionomias do campo limpo e campo sujo. No entanto, exceto pela inversão na prevalência de hemicriptófitas e de caméfitas nessas fitofisionomias, os padrões encontrados na EESB são semelhantes aos observados na Estação Ecologia e Experimental de Itirapina, SP, e em outras áreas de Cerrado (BATALHA e MARTINS, 2004).
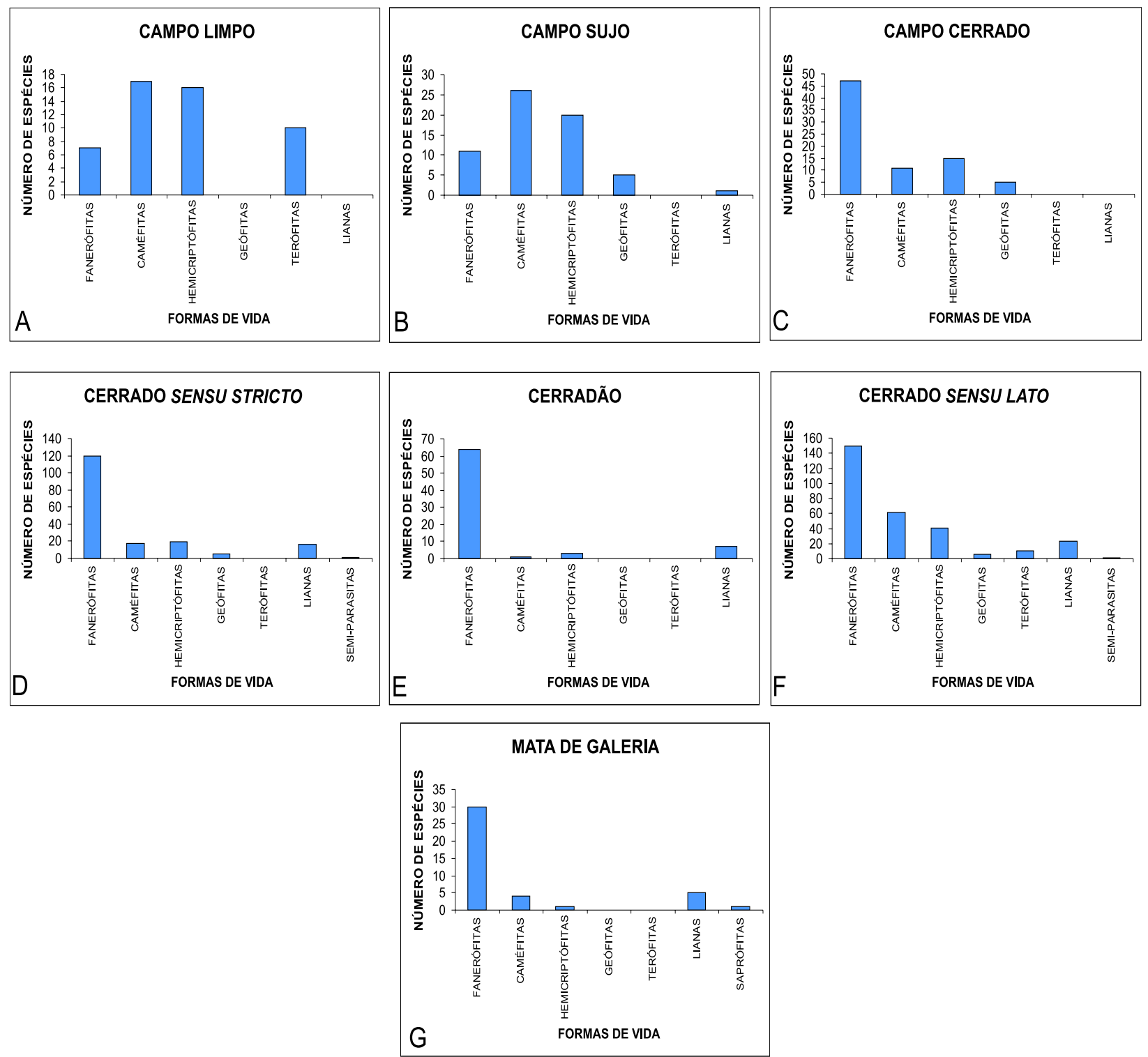

Figura 2 - Espectros biológicos das diferentes fitofisionomias de Cerrado e de Mata de Galeria na Estação Ecológica de Santa Bárbara (EESB), Estado de São Paulo.

Figure 2 - Biological spectra of different Cerrado physiognomies and Gallery Forest in Santa Bárbara Ecological Station (EESB), São Paulo State, Brazil.

R. Árvore, Viçosa-MG, v.31, n.5, p.907-922, 2007 
A forma de vida geofítica ocorreu apenas nas fisionomias intermediárias do Cerrado da EESB, o Cerrado sensu stricto, o campo cerrado e o campo sujo. A ausência das geófitas no Cerradão, como também em Mata de Galeria, deve-se, possivelmente, à exigência de níveis elevados de luminosidade por essas espécies. No campo limpo da EESB, que é intermitentemente alagado, as geófitas também não ocorreram, pois as condições pedológicas de encharcamento na maior parte do ano são muito diferentes daquelas encontradas nas formas fisionômicas intermediárias de Cerrado, em que os horizontes superficiais do solo são bem-drenados. Assim, as geófitas dificilmente serão encontradas nos campos limpos úmidos do domínio de Cerrado, pela pouca adaptabilidade dessa forma de vida aos alagamentos intermitentes.

\section{CONCLUSÕES}

O número de espécies encontradas nas fisionomias de Cerrado sensu lato na Estação Ecológica de Santa Bárbara constitui expressiva riqueza da vegetação de Cerrado.

As famílias mais ricas do Cerrado sensu lato nos levantamentos conjuntos de flora lenhosa e flora herbáceo-subarbustiva realizados na EESB são Asteraceae e Fabaceae. As duas famílias, juntamente com Poaceae, constituem um padrão florístico encontrado no Cerrado como um todo, conforme indicam este estudo e outros. Futuros levantamentos das duas floras são necessários para corroborações e verificação da extensão desse padrão.

Os espectros biológicos das diferentes fisionomias de Cerrado na EESB mostraram tendência de aumento no número de espécies das formas de vida fanerofítica e das lianas, e o decréscimo no número de espécies das formas de vida camefítica, hemicriptofítica e terofítica, quando se analisou a seqüência fitofisionômica no sentido do campo limpo para o cerradão e Mata de Galeria, corroborando estudos anteriores.

A predominância das caméfitas no campo limpo e o campo sujo pode ser efeito da proteção da EESB contra incêndios. Exceto por essa inversão na prevalência de hemicriptófitas e de caméfitas, os padrões encontrados na EESB são semelhantes aos verificados em outras áreas de Cerrado.

A forma de vida geofítica ocorreu apenas nas fisionomias savânicas intermediárias, não sendo encontrada no campo limpo da EESB, que é intermitentemente úmido.

\section{AGRADECIMENTOS}

Os autores agradecem às seguintes intituições e pessoas: Instituto Florestal do Estado de São Paulo, pelo apoio logístico e permissão para o estudo; FAPESP, pelo financiamento; funcionários da EESB, pelo apoio nos trabalhos de campo e colaboração; professores Hermógenes de Freitas Leitão Filho, João Semir Jorge e Jorge Yoshio Tamashiro, pela colaboração na identificação do material botânico; aos demais professores, aos funcionários do Departamento de Botânica da UNICAMP e aos colegas da Pós-Graduação em Biologia Vegetal da UNICAMP pelo apoio e amizade. Agradecem, ainda, aos revisores do artigo pela revisão atenta que em muito melhorou a qualidade do texto.

\section{REFERÊNCIAS}

ANGIOSPERM PHYLOGENY GROUP-APG. An update of the angiosperm phylogeny group classification for the orders and families of flowering plants: APG II. Botanical Journal of the Linnean Society, v.141, p.399-436, 2003.

BARRETO, F. V. B. Áreas naturais do estado de São Paulo. São Paulo: Conselho Estadual do Meio Ambiente, 1985. 16p.

BATALHA, M. A.; MANTOVANI, W. Reproductive phenological patterns of cerrado plant species at the Pé-de-Gigante Reserve (Santa Rita do Passa Quatro, SP, Brazil): a comparison between the herbaceous and woody floras. Revista Brasileira de Biologia, v.60, n.1, p.129-145, 2000.

BATALHA, M. A.; MANTOVANI, W. Floristic composition of the cerrado in the Pé-de-Gigante Reserve (Santa Rita do Passa Quatro, southeastern Brazil). Acta Botanica Brasílica, v.15, n.3, p.289-304, 2001.

BATALHA, M. A.; MARTINS, F. R. The vascular flora of Cerrado in Emas National Park (Goiás, Central Brazil). SIDA, v.20, n.1, p.295-311, 2002.

BATALHA, M. A.; MARTINS, F. R. Floristic, frequency, and vegetation life-form spectra of a cerrado site. Brazilian Journal of Biology., v.64, n.2, p.201-209, 2004.

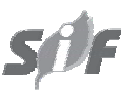

R. Árvore, Viçosa-MG, v.31, n.5, p.907-922, 2007 
CASTRO, A. J. F. et al. How rich is the flora of Brazilian Cerrados? Annals of the Missouri Botanical Garden, v.86, p.192-224, 1999.

COUTINHO, L.M. O conceito de cerrado. Revista Brasileira de Botânica, v.1, n.1, p.17-23, 1978.

COUTINHO, L.M. O bioma do Cerrado. In: KLEIN, A. L. (Org.). Eugen Warming e o Cerrado brasileiro um século depois. São Paulo:

Universidade Estadual de São Paulo, 2002. p.77-91.

EITEN,G. Vegetação do cerrado. In: PINTO, M. N. (Ed.). Cerrado: caracterização, ocupação e perspectiva. Brasília: Universidade de Brasília, 1990. p.9-65.

\section{EMPRESA BRASILEIRA DE PESQUISA} AGROPECUÁRIA - EMBRAPA. Sistema brasileiro de classificação dos solos. Rio de Janeiro: Centro Nacional de Pesquisa de Solos, 1999. 412p.

FILGUEIRAS, T. Herbaceous plant communities. In: OLIVEIRA, P. S.; MARQUIS, R. J. (Eds.). The cerrados of Brazil: Ecology and natural history of a neotropical savanna. New York: Columbia University Press, 2002.p.121-139.

GOODLAND, R.J.A. A physiognomic analisys of the "cerrado vegetation" of Central Brazil. Journal of Ecology, v.59, p.411-419, 1971.

MANTOVAni, W. Análise florística e fitossociológica do estrato herbáceosubarbustivo no cerrado na Reserva Biológica de Moji Guaçu e em Itirapina, SP. 1987. 213f. Tese (Doutorado em Ecologia).- Universidade Estadual de Campinas, Campinas, 1987.

MANTOVANI, W.; MARTINS, F.R. Florística do cerrado da Reserva Biológica de Moji-Guaçu, SP. Acta Botanica Brasílica, v.7, n.1, p.33-60, 1993.

R. Árvore, Viçosa-MG, v.31, n.5, p.907-922, 2007
MEIRA NETO, J. A. A. \& MARTINS, F. R. Estrutura do sub-bosque herbáceo-arbustivo da mata da silvicultura, uma floresta estacional semidecidual nomunicípio de Viçosa-MG. Revista Árvore, v.27, n.4, p.459-471, 2003.

MEIRA NETO, J. A. A. et al. Composição florística, espectro biológico e fitofisionomia da vegetação de muçununga nos municípios de Caravelas e Mucuri, Bahia. Revista Árvore, v.29, n.1, p.139-150, 2005

MENDONÇA, R. C. et al. Flora Vascular do Cerrado. In: SANO, S. M.; ALMEIDA, S. P. Cerrado: ambiente e flora. Brasília: Embrapa Cerrados, 1998 p. 289-556.

MOREIRA, A. G. Effects of fire protection on savanna structure in Central Brazil. Journal of Biogeography, v.27, p.1021-1029, 2000.

MUELLER-DOMBOIS, D.; ELLENBERG, H. Aims and methods of vegetation ecology. New York: John Wiley \& Sons, 1974. 547p.

MYERS, N. et al. Biodiversity hotspots for conservation priorities. Nature, v.403, p.853-858, 2000.

RIZZINI, C.T. A flora do cerrado. Análise florística das savanas centrais. In: FERRI, M. G. (Coord.). Simpósio sobre o cerrado. São Paulo: Universidade de São Paulo, 1963. p.125-178.

SETZER, J. Atlas climático e ecológico do Estado de São Paulo. São Paulo: Comissão Interestadual da Bacia do Paraná, Uruguai e Centrais Elétricas de São Paulo,. 1966. 35p.

VENTURA, A.; BEREENGUT, A.; VICTOR, M.A.M. Características edafo-climáticas das dependências do Serviço Florestal do Estado de São Paulo. Silvicultura em São Paulo, v.41, p.57-140, 1965/1966.

WARMING, E. Lagoa Santa. In: WARMING, E.; FERRI. M. G. Lagoa Santa; a vegetação de cerrados brasileiros. São Paulo: Edusp/Itatiaia 1973. p.1-284. 